\title{
Implantatentfernung nach Frakturversorgung oder Osteotomie am Knie (Tipps und Tricks)
}

\author{
Tobias C. Drenck, Achim Preiss, Ralph Akoto, Karl-Heinz Frosch
}

\section{Zusammenfassung}

Nach erfolgreicher osteosynthetischer Versorgung stellt sich grundsätzlich die Frage nach der Materialentfernung. Technische Schwierigkeiten finden sich je nach Literatur in bis zu 20\% der Fälle. Es muss deshalb geklärt werden, welches Material entfernt werden sollte. Eine generelle Empfehlung zur Materialentfernung gibt es nicht, die Indikation ist jeweils individuell in Abhängigkeit von anatomischer Lokalisation, Beschwerdebild, Alter und Grunderkrankungen des Patienten sowie Art des Materials zu stellen. Bei der Metallentfernung können durchaus Probleme auftreten, welche meist durch zerstörte Schrauben oder Nagel-Extraktions-Interfaces verursacht werden. Abgebrochene oder kalt verschweißte Schrauben können den Eingriff dabei erheblich erschweren. Bei der Entfernung ist darauf zu achten, dass das spezifische, für das Material vorgesehene Instrumentarium vorhanden ist. Auch mit auftretenden Problemen sollte gerechnet werden und ein entsprechendes „Rescue Set“ zur Verfügung stehen. Bereits beim Einbringen des Materials sollte sorgfältig vorgegangen werden und eine später notwendige Materialentfernung berücksichtigt werden.

\section{Implant Removal after Osteosyn- thesis or Osteotomy of the Knee}

Every successful osteosynthesis brings up the question whether the implant should be removed. Technical difficulties related to implant removal are described in up to $20 \%$ of the cases depending on the literature. The main question is, which implants have to be removed. There is no general recommendation for implant removal. The indication depends on localization, complaints by the patient, age and comorbidities as well as the type of implant. Problems in the operation itself are usually due to damaged screws or nail interfaces. Broken or cold welded screws are some reasons the implant removal becomes more difficult than expected. It is advised to have the specific instruments for implant removal on hand as well as a "rescue set". The implant removal should always be considered while performing the initial osteosynthesis to make the implant removal successful.

\section{Allgemeines zur Materialentfernung}

Die Materialentfernung gehört zu den häufigsten Eingriffen am Skelettsystem in der Orthopädie und Unfallchirurgie, Müller et al. gehen von etwa 74000 Implantatentfernungen/Jahr und einer gesamtwirtschaftlichen Belastung von 183 Mio. $€$ in der BRD aus [3]. In bis zu

OP-JOURNAL 2016; 32: 138-144

(c) Georg Thieme Verlag KG Stuttgart · New York DOI http://dx.doi.org/10.1055/s-0042-116193
60\% der Fälle handelt es sich um elektive Operationen bei beschwerdefreien Patienten, daher müssen sowohl an die Indikationsstellung als auch an die Durchführung hohe Ansprüche gestellt werden. Evidenzbasierte Empfehlungen zur Indikation von Materialentfernungen sind in der Literatur selten [4]. Eine gute präoperative Planung und das Berücksichtigen spezieller intraoperativer Tipps und Tricks können für das Gelingen entscheidend sein. Vielfach wird eine Materialentfernung von Patienten gewünscht oder Ärzten empfohlen. Nicht selten können dabei aber erhebliche Probleme und auch Komplikationen auftreten. Hinsichtlich der Indikation ist deshalb genau zu prüfen und abzuwägen, ob die Materialentfernung sinnvoll, notwendig und nützlich für den Patienten ist. Auch scheinbar einfache Materialentfernungen sollten gut vorbereitet sein; es muss immer damit gerechnet werden, dass die Schraubenköpfe beschädigt sind. Insbesondere bei Titanimplantaten kann eine Kaltverschweißung der Schrauben vorliegen. Außerdem können Vernarbungen und Verknöcherungen den Zugang zum Implantat erschweren. Das entsprechende Instrumentarium zur Entfernung des Implantats, aber auch ein entsprechendes „Rescue Set“ für die Entfernung von abgebrochenen oder abgedrehten Schrauben sollte ebenfalls zur Hand sein. Bereits beim Einbringen des Materials sollte der Operateur deshalb immer auch die evtl. notwendige Materialentfernung im Hinterkopf haben und entsprechende Vorkehrungen treffen. An einigen Körperregionen ist die Materialentfernung durch die Nähe zu neurovaskulären Strukturen mit hohen Komplikationsraten behaftet (z.B. posterolateraler Tibiakopf) und sollte deshalb möglichst vermieden werden. Bei anderen Regionen hingegen profitieren die Patienten nicht selten von einer Materialentfernung, was zu reduzierten Schmerzen und verbesserter Beweglichkeit führt (z.B. lateraler Tibiakopf). Das Vorgehen ist immer individuell mit dem Patienten abzustimmen. Häufig versteht der Patient die Metallentfernung als Abschluss der Frakturbehandlung, eine generelle Empfehlung zur Materialentfernung kann jedoch nicht gegeben werden [5].

\section{Indikation}

Bei jeder Metallentfernung handelt es sich um einen erneuten operativen Eingriff mit den üblichen operativen Risiken; die Materialentfernung muss daher im Verhältnis zum therapeutischen Nutzen stehen. Auch das dauerhafte Belas- 
sen eines Implantats kann durch biomechanische und biologische Reaktionen Spätkomplikationen verursachen. So führt die biomechanische Interaktion zu einem Strukturumbau im Knochen, der diesen schwächen kann. In statisch belasteten Röhrenknochen vermindert das Implantat die Elastizität des Knochens [3]. Diskutiert werden auch die Möglichkeiten von Spätinfekten und Metallunverträglichkeiten, wobei Letztere durch die häufigere Verwendung von Titanimplantaten selten geworden ist. Die Prävalenz für Nickel- und Chromallergien liegt jedoch bei 10-15\% [6]. Teilweise wird auch das Risiko einer Kanzerogenität diskutiert, bisher gibt es jedoch diesbez. in der Literatur keinen eindeutigen Hinweis. In einer schwedischen Studie von 117000 Patienten nach Implantation einer Endoprothese ergab sich kein generell erhöhtes Risiko für Tumoren [6]. Zudem können insbesondere nach Osteosynthesen am Tibiakopf diagnostische Maßnahmen wie MRT- oder CT-Bildgebung durch Artefakte deutlich erschwert werden. Ferner machen Implantate im Bereich des Kniegelenks eine endoprothetische Versorgung ungleich schwerer und erfordern in den meisten Fällen eine Entfernung des Materials [1].

Häufigster Grund einer Metallentfernung sind störende Implantate.

Ein störendes Implantat ist eine der häufigsten Indikationen zur Metallentfernung [1]. Jedoch können auch korrekt einliegende Implantate bei Frakturen mit Gelenkbeteiligung fälschlicherweise für Beschwerden verantwortlich gemacht, da die Beschwerden z.B. durch eine beginnende Arthrose verursacht werden. Andernfalls können etwa am medialen Schienbeinkopf, durch den geringen Weichteilmantel, überstehende Schraubenspitzen massive Beschwerden verursachen. Ein weiteres Beispiel ist ein proximal nur leicht überstehender Tibianagel, der häufig Beschwerden im Bereich des Hoffa-Fettkörpers und des Lig. patellae verursacht. Überwiegend einig sind sich die meisten Autoren, dass Implantate im Wachstumsalter entfernt werden sollten.

\section{Bildgebung}

Vor jeder Metallentfernung muss sich der Operateur vergewissern, dass die Fraktur oder Osteotomie konsolidiert ist.

Vor jeder Materialentfernung sollte sich der Operateur vergewissern, dass eine
Fraktur oder Osteotomie ausreichend konsolidiert ist. Pseudarthrosen kommen im Bereich der unteren Extremität am häufigsten vor, die Tibia ist der am meisten betroffene Knochen. In einer Studie von 392 Patienten mit einer Pseudarthrose fanden sich 216 davon im Bereich des Unterschenkels und 65 am Oberschenkel. Pseudarthrosen langer Röhrenknochen sind bei Plattenosteosynthesen häufiger als bei Marknagelosteosynthesen [7]. Zur Beurteilung sind immer aktuelle Röntgenaufnahmen in 2 Ebenen durchzuführen, die Implantate müssen hier vollständig dargestellt werden. Entscheidend für die Beurteilung ist, dass die verheilte Osteotomie bzw. Fraktur korrekt in 2 senkrecht zueinander stehenden Ebenen abgebildet und beurteilt werden kann und nicht das Osteosynthesematerial. Liegt der ehemalige Frakturbereich nicht im zentralen Strahlengang, wie etwa bei Nagelungen langer Röhrenknochen, und kann daher nicht ausreichend beurteilt werden, müssen ggf. zusätzliche Aufnahmen durchgeführt werden. Ist eine Konsolidierung in konventionellen Röntgenaufnahmen nicht ausreichend zu beurteilen, muss gelegentlich zusätzlich ein CT durchgeführt werden; von einer regelhaften Durchführung einer CT-Bildgebung ist jedoch aufgrund der Strahlenbelastung abzuraten. Ist eine ausreichende Konsolidierung sichergestellt, gilt es, das Implantat radiologisch genau zu beurteilen. Abgebrochene oder verbogene Schrauben sind im konventionellen Röntgen häufig nur durch leichte Achsfehlstellungen festzustellen und sind projektionsbedingt in einigen Ebenen oft gar nicht zu erkennen. Lässt sich bereits präoperativ erkennen, dass eine gebrochene Schraube oder ein gebrochener Verriegelungsbolzen nur mit großem Aufwand entfernt werden kann, muss dies bereits in der präoperativen Planung berücksichtigt und mit dem Patienten besprochen werden [1].

\section{Plattenosteosynthesen}

Im Bereich des Kniegelenks kommen winkelstabilen Platten sowohl bei Versorgung der Tibiakopffrakturen als auch bei Osteotomien des Femurs und der Tibia eine bedeutende Rolle zu. Wie bei jedem winkelstabilen Implantat sollte man auch hier bei der Entnahme auf kalt verschweißste Schrauben vorbereitet sein.

Einen hohen Schwierigkeitsgrad stellen sicherlich mittels Plattenosteosynthese versorgte Tibiakopffrakturen dar. Kom- plexe Frakturmuster mit Gelenkbeteilung machen häufig eine kombinierte Versorgung über mehrere operative $\mathrm{Zu}$ gänge erforderlich. Insbesondere Frakturen der dorsalen Anteile des Tibiaplateaus müssen häufig über posteriore Zugänge adressiert werden. Hierzu ist lateralseitig meist eine Darstellung des N. peroneus am posterioren Rand des M. biceps femoris erforderlich [8]. Bei einer Metallentfernung ist das Auffinden und Darstellen durch Narbengewebe dann zusätzlich erschwert. Beim posterioren Zugang befinden sich die A. und V. poplitea in unmittelbarer Nähe; es empfiehlt sich daher ohne Blutsperre $\mathrm{zu}$ arbeiten, um die Gefäße palpieren zu können [9]. Durch die Nähe zu Gefäßund Nervenstrukturen gehören Materialentfernungen am dorsalen Tibiakopf sicherlich zu den technisch schwierigsten (Abb. 1). Eine Entfernung dorsal anliegender Platten sollte daher keinesfalls routinemäßig erfolgen. Auch Osteosynthesen im Bereich der Patella führen oft zu Beschwerden beim Hinknien und werden daher ebenfalls häufig entfernt (Abb.2). Auch einzelne überstehende Schrauben können insbesondere bei intraartikulärer Lage zu erheblichen Problemen führen und bedürfen einer umgehenden Materialentfernung (Abb. 4).

Trotz guter Osteosynthesen entwickeln jedoch bis zu 44\% der Patienten nach einer Tibiakopffraktur eine Gonarthrose; eine geplante endoprothetische Versorgung macht dann häufig eine Materialentfernung erforderlich.

\section{Materialentfernungen nach knienahen Osteotomien}

Die Materialentfernung nach Osteotomie stellt eine Besonderheit dar, es handelt sich meist um jüngere Patienten und elektive Operationen. Osteotomien zur Therapie von Achsdeformitäten bei unilateralen Arthrosen werden meist bei Patienten < 55 Jahren durchgeführt, Patienten bei denen zur Behandlung eines Maltrackings der Patella eine Torsionsosteotomie durchgeführt wird, sind meist deutlich jünger (Abb. 3). Das junge Alter der Patienten ist sicherlich ein Grund, weshalb Materialentfernungen nach Osteotomien in dieser Patientengruppe relativ häufig sind. Eine weitere Besonderheit stellt die Wahl der operativen Zugänge dar. In der Traumatologie bestimmt meist das zu versorgende Frakturmuster den operativen Zugang. Bei Osteotomien im Bereich des Femurs oder der Tibia werden operative Zugänge 

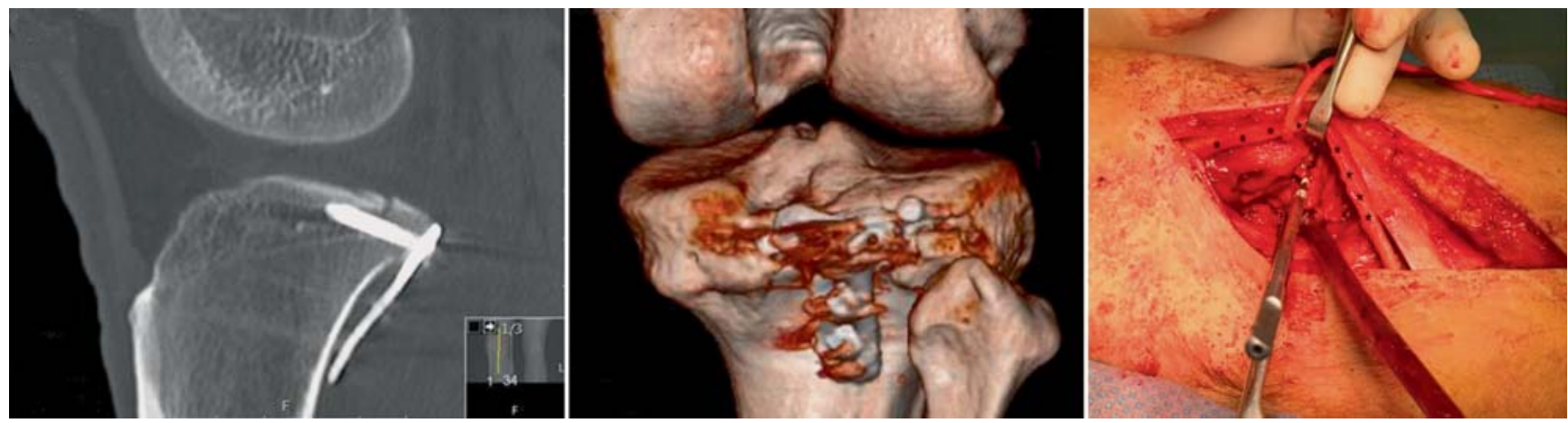

Abb. 1 Eine Osteosynthese im Bereich des posterolateralen Tibiaplateaus durch kanülierte Schrauben und zusätzliche Abstützung mittels T-Platte. Der OP-Zugang erfordert die Darstellung des N. peroneus $\left({ }^{*}\right)$. Im Falle einer Materialentfernung kann die Anatomie durch Vernarbungen unübersichtlich sein. Um den Nerv nicht zu verletzen, ist eine Entfernung hier nur in zwingenden Fällen angeraten.
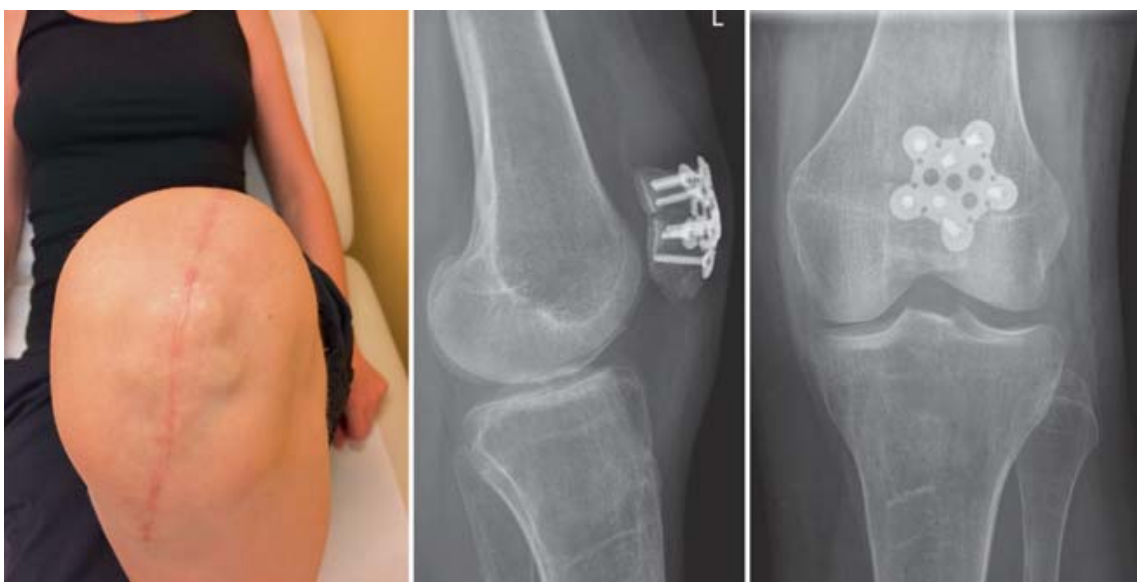

Abb. 2 Eine junge Patienten nach Pseudarthrose einer Patellafraktur, mittlerweile mit Patellaplatte versorgt, welche zum Ausheilen der Pseudarthrose führte. In der Regel ist eine Materialentfernung nach Patellafraktur nicht notwendig. In seltenen Fällen kann die Platte dennoch, wie bei dieser Patientin, stören, sodass die Materialentfernung empfohlen werden kann. Zuggurtungsosteosynthesen hingegen erfordern i. d. R. eine Materialentfernung 6-9 Monate postoperativ, da die eingebrachten Drähte fast immer von den Patienten als störend empfunden werden.

gewählt, die eine besondere Schonung der Strukturen erlauben. Eine Osteotomie des distalen Femurs wird unterhalb des M. vastuts medialis oder lateralis durchgeführt. Bei dem Zugang von medial ist darauf zu achten, die Strukturen dorsal der Linea aspera durch einen eingebrachten Hohmann-Haken sicher zu schützen. Bei der Entfernung von Platten des medialen Schienbeinkopfs sollte darauf geachtet werden, keine größeren Anteile des oberflächlichen ventralen Innenbandansatzes zu verletzen, die Übersicht über die Platte ist jedoch gut, und Gefäß-Nerven-Strukturen befinden sich nicht in unmittelbarer Nähe. Nach Osteotomien im Bereich des Kniegelenks werden von Patienten häufig Beschwerden angegeben, Niemeyer et al. zeigten, dass 40,6\% aller Patienten nach hoher tibialer Umstellungsosteotomie (HTO) mehr oder weniger über Beschwerden im Bereich des Plattenlagers klagten [10]. Bei distalen Femurosteotomien mit lateraler Plattenlage ist diese Rate nach unserer Erfahrung noch deutlich höher! Hier kommt es in einem hohen Prozentsatz zu Irritationen am Tractus iliotibialis. Eine Materialentfernung ist an dieser Lokalisation deshalb oft notwendig. Deutlich weniger irritierend ist das Osteosynthesematerial auf der Innenseite, weshalb die von medial geführten Osteotomien am distalen Femur zunehmend eine Rolle spielen. Insbesondere bei öffnenden Osteotomien muss vor der Materialentfernung eine sichere Durchbauung festgestellt werden, je nach Literatur liegt das Risiko für eine Pseudarthrose hier bei ca. $5 \%$. Nikotinkonsum, eine intraoperative Fraktur der Gegenkortikalis und Übergewicht sind hier als Risikofaktoren zu nennen, im Zweifel muss eine CT durchgeführt werden [11].

\section{Intramedulläre Stabilisierung}

Eine routinemäßige Entfernung intramedullärer Implantate, insbesondere bei Beschwerdefreiheit ist beim Erwachsenen nicht gerechtfertigt.

Die intramedulläre Stabilisierung stellt die bevorzugte Methode zur Versorgung von Femur- und Tibiaschaftfrakturen dar. Über einen kleinen operativen $\mathrm{Zu}-$ gang lässt sich so häufig eine belastungsstabile Reposition und Osteosynthese erreichen. Weniger eindeutig ist die Literatur jedoch hinsichtlich der Notwendigkeit einer Materialentfernung intramedullärer Kraftträger. Nach Durchbauung der Fraktur, meist 1-2 Jahre nach Stabilisierung, wurde früher auch bei asymptomatischen Patienten fast regelhaft eine Materialentfernung durchgeführt. Auch die sozioökonomischen Auswirkungen einer routinemäßigen Entfernung sind nicht unerheblich; Boerger et al. zeigten, dass die Entfernung eines Marknagels durchschnittlich zu einer Arbeitsunfähigkeit von 11 Tagen führt [12]. Entscheidend für eine erfolgreiche Entfernung eines Marknagels ist eine saubere Arretierung der Ausschlaginstrumente im Marknagel, da der Nagel im Gegensatz zur Platte durch seine intramedulläre Lage kaum für Manipulationen zugänglich ist. Ein bei Implantation zerstörtes Interface am Marknagel kann eine Materialentfernung schwierig gestalten (Abb. 10). Entscheidend ist zudem, das Gewinde für das Ausschlaginstrument sorgfältig von Weichteilen oder knöchernen Anbauten $\mathrm{zu}$ befreien. Erschwert wird dies, da insbesondere beim proximalen Femurnagel die direkte Sicht auf das Interface fehlt. Eine gravierende intraoperative Komplikation ist eine iatrogene Fraktur, zu der es beim Ausschlagen des Nagels kommen kann. Verschiedene Autoren berichten von entsprechenden 


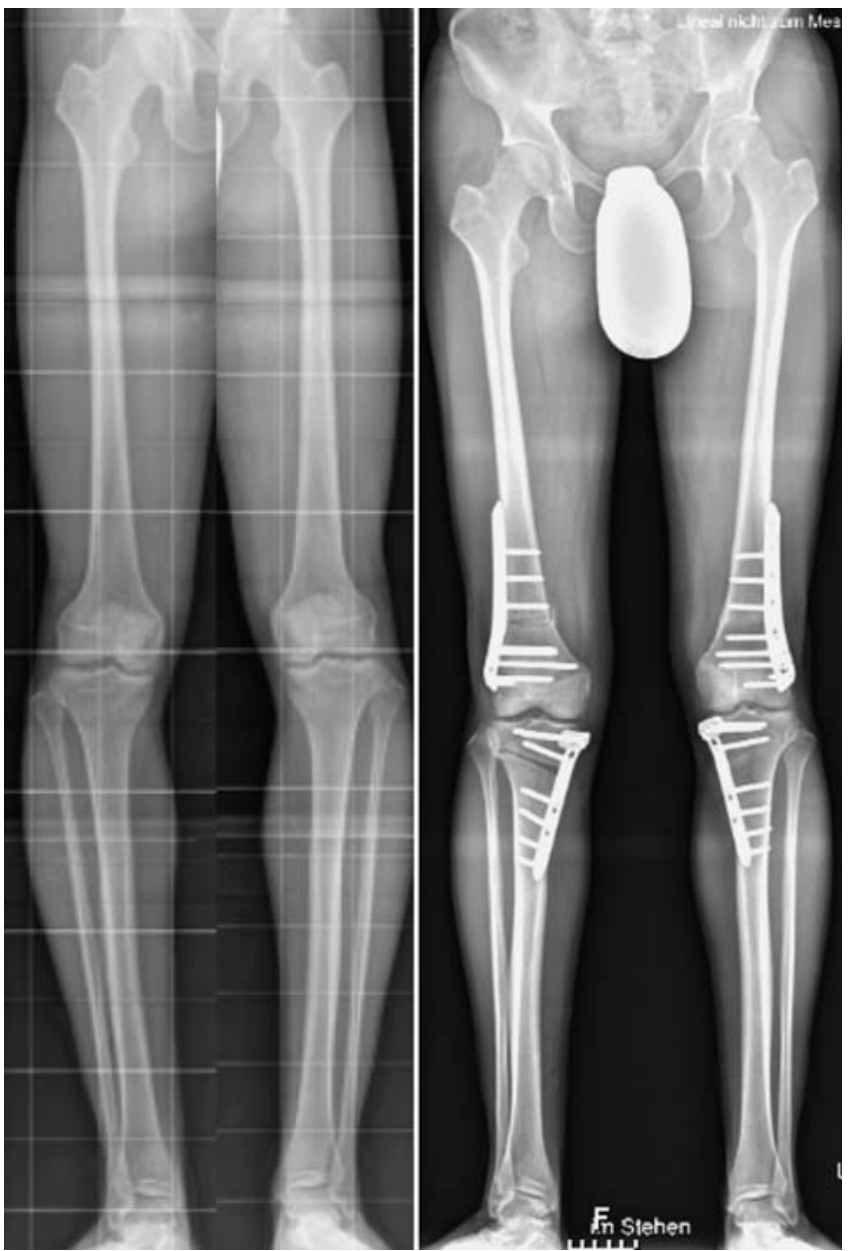

Abb. 3 Die Abbildung zeigt einen jungen Patienten, bei dem zur Behandlung eines symptomatischen „inwardly pointing knee" eine kombinierte Torsionsosteotomie des distalen Femurs sowie eine valgisierende Open-WedgeOsteotomie der proximalen Tibia erfolgte. Sechs Monate postoperativ ist die Osteotomie links komplett durchbaut und der Patient annährend beschwerdefrei. Aufgrund von Druckschmerzen im Bereich des Plattenlagers, insbesondere am lateralen distalen Femur, ist eine Materialentfernung empfehlenswert. Die Osteotomien am rechten Bein sind nach 4 Wochen noch nicht durchbaut.
Komplikationen in 6-21\% der Fälle bei der Marknagelentfernung älterer Systeme. Wurden derartige Komplikationen früher häufiger beobachtet, scheinen sie bei modernen Systemen seltener zu sein: In einer Untersuchung von Hora et al. kam es bei 460 entfernten Marknägeln in einem Zeitraum von 11 Jahren nur zu einer Fraktur. Es finden sich jedoch Berichte, bei denen es durch das akzidentelle Belassen einer Verriegelungsschraube beim Ausschlagen des Implantats zu einer Fraktur kam, und bei der Entfernung eines retrograden Femurnagels durch das ungenügende Entfernen knöcherner Überbauungen zu einer kondylären Abscherfraktur kam [4].

\section{Tibiamarknagel}

Symptomatische Patienten nach Tibiamarknagelung klagen meist über einen vorderen Knieschmerz.

Typische Beschwerden nach Tibiamarknagelung sind der vordere Knieschmerz und Schmerzen im Bereich der Verriegelungsbolzen. Hinsichtlich einer Verbesserung der Symptome finden sich in der
Literatur teilweise sehr unterschiedliche Ergebnisse. In einer Studie von Keating et al. führte die Entfernung eines Tibianagels in 45\% der Fälle zu einer kompletten Schmerzfreiheit, in 35\% der Fälle führte die Entfernung zu einer Verbesserung, wohingegen bei $20 \%$ der Patienten auch weiterhin die Beschwerden bestanden. Court-Brown et al. stellten bei 97\% der Patienten eine Verbesserung der Beschwerden fest, jedoch gaben hier ca. 3\% der Patienten an, dass die Beschwerden nach der Entfernung stärker wurden. Die Arbeitsgruppe von Krettek et al. stellte in einer Analyse von $44 \mathrm{~Pa}$ tienten fest, dass sich die Symptome nach Entfernung in 73\% der Fälle verbesserten, in $8 \%$ kam es jedoch zu einer Verschlechterung. Außerdem zeigten 17\% der zuvor 18 asymptomatischen Patienten neu aufgetretene Beschwerden nach Entfernung des Marknagels [13].

\section{Femurmarknagel}

Auch die Auswirkungen der Entfernung eines Femurmarknagels auf die Beschwerden des Patienten lassen sich ähnlich dem Tibianagel nur schwer ab- schätzen. Gosling et al. analysierten 164 Patienten, von denen 53\% Beschwerden zeigten. Nach Explantation gaben 78\% eine Besserung der Beschwerden an, 7\% klagten über eine Verschlechterung. Jedoch klagen auch hier $20 \%$ der präoperativ asymptomatischen Patienten über neu aufgetretene und anhaltende Beschwerden nach der Explantation.

\section{Allgemeine Tipps und Tricks zur Materialentfernung}

Häufig sind Materialentfernungen die ersten operativen Eingriffe in der Weiterbildung zum Facharzt für Orthopädie und Unfallchirurgie. Auf diese Weise können die operativen Zugänge ohne die erforderliche anspruchsvolle Reposition oder Osteotomie des Primäreingriffs erlernt werden. Dies soll jedoch keinesfalls suggerieren, dass es sich bei Materialentfernungen generell um unkomplizierte Operationen handelt.

\section{Prävention}

Eine erfolgreiche Materialentfernung beginnt bereits bei der Implantation und sollte von jedem Operateur berücksichtigt werden. Schwarz et al. stellten in einer Multicenterstudie zur Entfernung von LCP-Titanplatten fest, dass es bei 20\% der Materialentfernungen zu technischen Problemen kam. Die mit Abstand häufigste Komplikation betrifft dabei den Antrieb der Schrauben [14] (Abb. 5). Folgende Punkte sollten daher bereits bei der Implantation beachtet werden [15]:

- Nur unbeschädigte Schraubendreher verwenden, abgerundete Kanten können den Schraubenantrieb beschädigen und die Kraft nicht richtig übertragen. Ist der Schraubenantrieb erst zerstört, lässt sich die Schraube meist auch mit einem neuwertigen Schraubendreher nicht mehr entfernen.

- Beim Einbringen von winkelstabilen Schrauben sollte dringend das richtige Drehmoment beachtet werden und der Drehmomentbegrenzer verwendet werden. Bei unkontrolliert festem Anziehen der Schrauben kann es zur sog. Kaltverschweißung kommen.

- Wird eine Schraube beim Hineindrehen beschädigt, sollte diese umgehend intraoperativ ausgetauscht werden.

- Bei winkelstabilen Schrauben sollte darauf geachtet werden, diese im korrekten Winkel einzudrehen (Abb.6). Sofern es sich nicht um polyaxiale Schrauben handelt, sollte die maximal zulässige Angulation beachtet werden. 


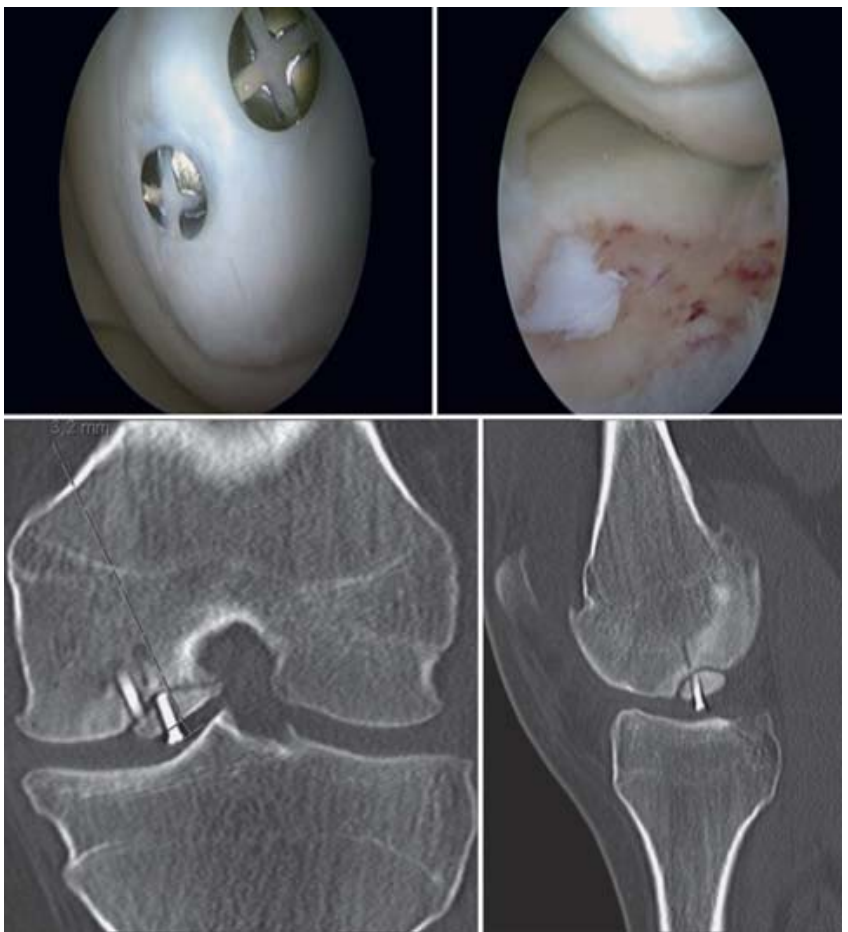

\section{Operationsbericht}

Vor jeder Materialentfernung sollte der Operationsbericht der Implantation gelesen werden.

Das Lesen des primären Operationsberichts der Implantation des Osteosynthesematerials ist für eine erfolgreiche Materialentfernung unerlässlich. Das verwendete Implantat, Schrauben und zusätzlich verwendete Unterlegscheiben müssen anhand des Operationsberichts genau zu identifizieren sein. Entscheidend ist dies bei der Vielzahl an verschiedenen auf dem Markt erhältlichen Implantaten und Schrauben, damit zur Materialentfernung das passende Extraktionsintrumentarium zur Verfügung steht. Schon beim Schreiben des Berichts sollte darauf geachtet werden, entscheidende Besonderheiten hinsichtlich einer Materialentfernung zu dokumentieren, etwa eine beim Eindrehen beschädigte oder gebrochene Schraube. Liegen Nerven oder Gefäße in unmittelbarer Nähe des Implantats, sollten die räumlichen Beziehungen zueinander aus dem Bericht hervorgehen. Wurde ein Patient außerhalb der eigenen Klinik operiert, sollte der Operationsbericht angefordert werden [1].
Abb. 4 Beispiel Schraubenlage: In diesem Fall stellte sich ein Patient nach auswärtiger Refixierung einer Osteochondrosis dissecans in unserer Sprechstunde vor. Die Schrauben stehen ins Gelenk und haben zu Knorpelschäden geführt. Die Schrauben konnten arthroskopisch entfernt werden. Eine intraartikuläre Schraubenlage erfordert i.d. R. eine Materialentfernung. einer intraartikulären

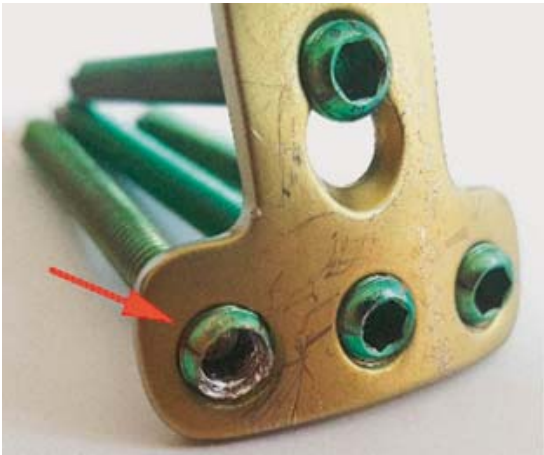

Abb. 5 Zerstörter Schraubenantrieb.

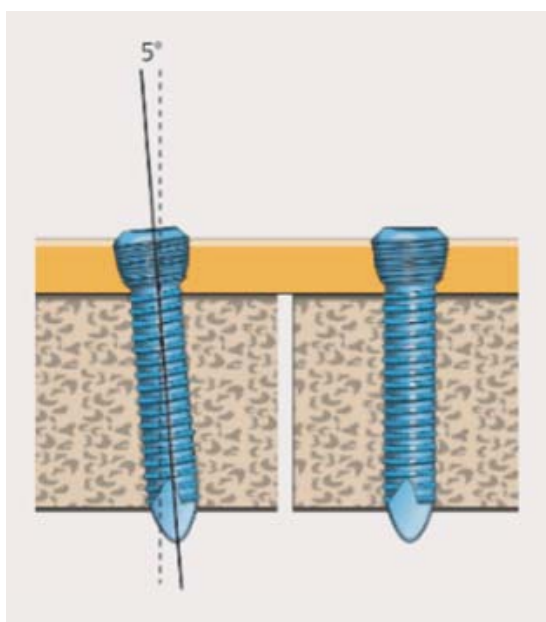

Abb. 6 Bei monoaxialen Schrauben ist darauf zu achten, dass diese genau rechtwinklig zur Platte gebohrt und eingeschraubt werden um das Verriegelungsgewinde nicht zu beschädigen [2].

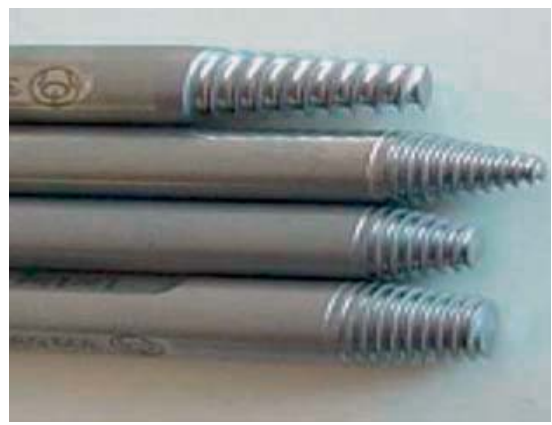

Abb. 7 Erstes Hilfsmittel bei zerstörtem Schraubenkopf sind linksschneidende Extraktionsschrauben, die in den Schraubenkopf eingedreht werden [2]. sein, den Inbus aufzubohren, da die Extraktionsschraube fasst. Die Extraktionsschraube muss genau passen und darf nicht zu klein gewählt werden (Abb. 8), im Zweifel kann an einer bereits entfernten Schraube gemessen werden.

- Gelingt eine Entfernung durch die Extraktionsschraube nicht, kann der Schraubenkopf durch Aufbohren vom Schaft getrennt werden (Abb.9). Für besonders harte Bohreinsätze gibt es Spezialbohrer. Die Platte kann anschließend entfernt werden. Der verbliebende Schaft der Schraube kann häufig gefasst und herausgedreht werden. Gelingt dies nicht, kann dieser mit einer linksdrehenden Hohlfräse überbohrt werden. In Kombination 


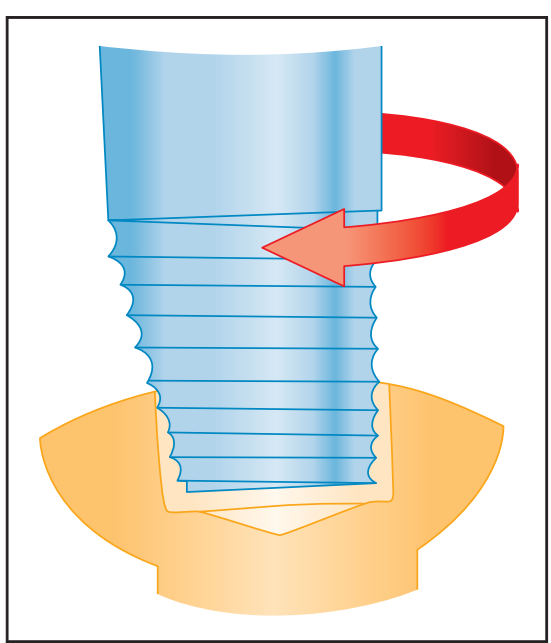

Abb. 8 Funktionsweise der linksdrehenden Extraktionschraube: durch Linksdrehung findet diese im zerstörten Schraubenkopf Halt [2].

mit einem linksschneidenden Innengewinde wird so der verbliebende Schaft entfernt.

- Vor dem Überbohren einer verbliebenden Schraube sollte stets eine Risiko-Nutzen-Abwägung erfolgen und nur angewandt werden, sofern hierdurch keine Frakturgefahr gegeben ist, im Zweifel wird das Material belassen.

- Auch die Entscheidung, zerstörte Anteile eine Osteosynthese zu belassen, kann eine gute Materialentfernung ausmachen, sofern der damit verbundene Schaden zu groß wäre.

\section{Postoperativ, Nachbehandlung und die Gefahr von Refrakturen}

Postoperativ erfolgt eine Röntgenkontrolle der erfolgreichen Materialentfernung in 2 Ebenen zur Dokumentation. Die Fraktur kann nun ohne Überlagerungen durch das Osteosynthesematerial beurteilt werden. Sofern intraoperativ von einer sicheren knöchernen Konsolidierung ausgegangen werden kann, eignet sich auch eine Dokumentation mittels Bildwandler am Ende der Operation, sofern die Aufnahmen anschließend archiviert werden können. In der Regel kann die untere Extremität nach einer Materialentfernung am Kniegelenk in vollem Umfang belastet und bewegt werden. Jedoch handelt es sich bei den zurückbleibenden Schraubenlöchern um Sollbruchstellen, sodass es sinnvoll sein kann, Spitzenbelastungen wie Kontaktsportarten für die ersten Monate zu vermeiden [1]; die meisten Refrakturen ereignen sich in den ersten 4 Monaten nach Implantatentfernung [4]. Biomechanische Untersuchungen zeigten, dass

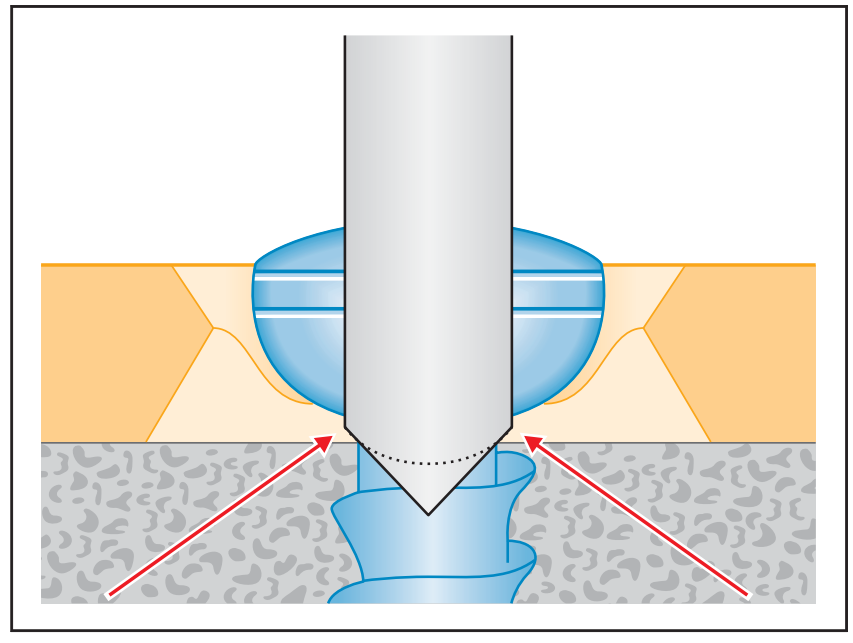

Abb. 9 Durch Aufbohren des Schraubenkopfs kann dieser vom Schaft getrennt werden [2].
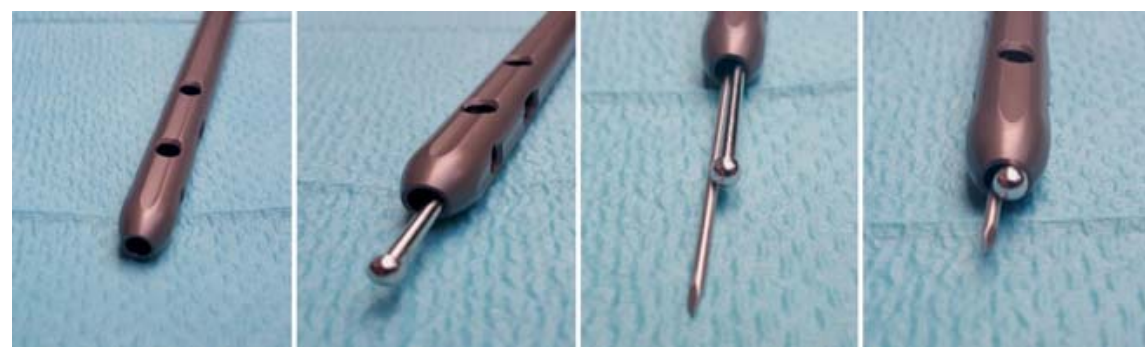

Abb. 10 Gezeigt wird eine Technik zum Entfernen von Marknägeln bei zerstörten ExtraktionsInterface oder bei gebrochenem Nagel: Zunächst wird ein Olivendraht nach distal durch den Nagel vorgeschoben, anschließend wird ein Führungsdraht entsprechender Größe parallel dazu eingebracht. Beim Zurückziehen des Olivendrahts verblockt sich dieser nun in der distalen Öffnung durch den Führungsdraht. Durch Aufbringen eines Spannfutters auf den Olivendraht kann der Nagel nun entfernt werden.

es in einem 2,8- und 3,6-mm-Schraubenloch zu einer 55\%igen Abnahme der Energieabsorption kommt [6].

Refrakturen nach Materialentfernungen stellen seltene, aber schwerwiegende Komplikationen dar (Abb. 11); Gras et al. geben eine Häufigkeit von $0-1,5 \%$ an. Im Bereich der Tibia wurde in 3 Studien mit insgesamt über 300 entfernten Tibiamarknägeln keine einzige Fraktur beobachtet. Leu et al. berichten von Refrakturen bei 2 von 194 Patienten nach einer Plattenentfernung an der Tibia [4]. Ein ähnliches Bild zeigt sich nach der Entfernung antegrader und retrograder Femurnägel, in 3 Studien mit insgesamt 293 Nagelentfernungen kam es zu keiner dokumentierten Refraktur. Nach der Entfernung von Plattenosteosynthesen im Bereich des distalen Femurs kam es in einer kleinen Studie von 15 Patienten bei 4 Patienten zu einer erneuten Fraktur in einem Zeitraum bis 10 Wochen [4].

\section{Schlussfolgerung}

- Eine erfolgreiche Materialentfernung beginnt mit der korrekten Implantation des Materials, das Anzugsdrehmoment winkelstabiler Schraubensysteme ist zwingend zu beachten.

- Die Indikation zur Entfernung sollte sorgfältig gestellt und mit dem Patienten besprochen werden. Eine generelle Empfehlung zur Materialentfernung gibt es nicht.

- Zur OP-Vorbereitung gehört die aktuelle Bildgebung und das Lesen des Operationsberichts, um auf evtl. Schwierigkeiten vorbereitet zu sein. Der besondere Verlauf von Nerven und Gefäßen oder beschädigtes Osteosynthesematerial sollte hier dokumentiert sein.

- Komplikationen im Rahmen von Materialentfernungen sind nicht selten, meist betrifft es das Schrauben-Interface. Entsprechende „Rescue“-Instrumente sollten bereitstehen und der Umgang damit vertraut sein. 


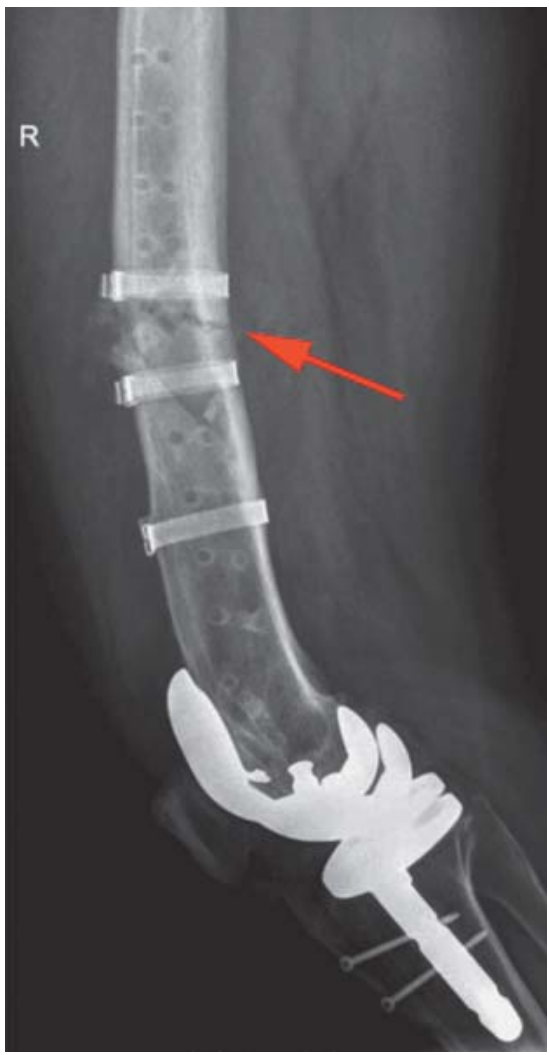

Abb. 11 Bei dieser 55-Jährigen kam es nach Implantation einer Knieendoprothese zu einer periprothetischen Fraktur und anschließender Osteosynthese mittels LISS-Platte. Nach zeitgerechter Materialentfernung kam es dann einige Monate später bei einem schnellen Richtungswechsel zur Refraktur (Pfeil). Die Gefahr von Refrakturen ist bei Plattenosteosynthesen höher als nach Marknagelung und tritt meist in den ersten 4 Monaten nach Entfernung des Osteosynthesematerials auf.

\section{Literatur}

${ }^{1}$ Müller-Färber J. Die Metallentfernung nach Osteosynthesen. Indikationen und Risiken. Orthopäde 2003; 32: 1039-1058

${ }^{2}$ Höntzsch $D$. Tipps und Tricks für die problematische Metallentfernung von winkelstabilen Platten-Schrauben-Systemen. OP-JOURNAL 2012; 28: 170-173

${ }^{3}$ Müller M, Mückley T, Hofmann GO. [Costs and complications of implant removal]. Trauma Berufskrankh 2007; 9: S297-S301

${ }^{4}$ Gras F, Marintschev I, Lenz M et al. [Fractures due to removal of support devices]. Trauma Berufskrankh 2014; 16: 341-348

${ }^{5}$ Müller-Färber J. Die Metallentfernung in der Unfallchirurgie. Unfallchirurg 2003; 106: 653-670

${ }^{6}$ Schildhauer TA. [Metal removal operations]. Trauma Berufskrankh 2007; 9: S292-S296

${ }^{7}$ Grechenig S, Pfeifer C, Krutsch W et al. [Complication management for failed bone fracture healing: pseudarthrosis]. Chirurg 2015; 86: 919-924

${ }^{8}$ Frosch $K H$, Balcarek $P$, Walde $T$ et al. A new posterolateral approach without fibula osteotomy for the treatment of tibial plateau fractures. J Orthop Trauma 2010; 24: 515-520

${ }^{9}$ Raschke M, Zantop T, Petersen W. [Fracture of the tibial head]. Chirurg 2007; 78: 11571171

${ }^{10}$ Niemeyer P, Schmal H, Hauschild O et al. Openwedge osteotomy using an internal plate fixator in patients with medial-compartment gonarthritis and varus malalignment: 3-year results with regard to preoperative arthro- scopic and radiographic findings. Arthroscopy 2010; 26 : 1607-1616

${ }^{11}$ Hinterwimmer S, Feucht MJ, Imhoff AB. [High tibial osteotomy for varus arthritis]. Arthroskopie 2012; 25: 184-194

12 Boerger TO, Patel G, Murphy JP. Is routine removal of intramedullary nails justified. Injury 1999; 30: 79-81

13 Krettek C, Mommsen P. [Implant removal after intramedullary osteosyntheses. Literature review, technical details, and tips and tricks]. Unfallchirurg 2012; 115: 299-314

${ }^{14}$ Schwarz N, Euler S, Schlittler M et al. Technical complications during removal of locking screws from locking compression plates: a prospective multicenter study. Eur J Trauma Emerg Surg 2013; 39: 339-344

${ }^{15}$ Höntzsch D, Stuby FM. [Removal of plates and screws. Tips and tricks for problematic cases]. Unfallchirurg 2012; 115: 291-298

\section{Dr. med. Tobias C. Drenck}

Assistenzarzt

\section{Dr. med. Achim Preiss}

Oberarzt

Dr. med. Ralph Akoto

Oberarzt

Prof. Dr. med. Karl-Heinz Frosch

Chefarzt

Abteilung Unfall- und Wiederherstellungschirurgie mit Sektion Knie- und Schulterchirurgie, Sporttraumatologie Chirurgisch-Traumatologisches Zentrum

ASKLEPIOS Klinik St. Georg

Lohmühlenstraße 5

20099 Hamburg

k.frosch@asklepios.com www.chirurgisch-traumatologischeszentrum.de 